\title{
A note on strongly dense matrices
}

\author{
Miroslav Fiedler ${ }^{1}$ • Frank J. Hall ${ }^{2}$
}

Received: 10 June 2015 / Revised: 25 September 2015 / Accepted: 5 October 2015 /

Published online: 20 October 2015

(C) Springer International Publishing AG 2015

\begin{abstract}
In this note, strongly dense matrices are defined and some basic properties of these matrices are obtained. In particular, it is shown that for nonnegative and Boolean matrices, the product of conformable strongly dense matrices is strongly dense. Structural characterizations are presented for the idempotent nonnegative strongly dense matrices, as well as for the idempotent Boolean strongly dense matrices with a full diagonal. Connections with generalized complementary basic matrices are made.
\end{abstract}

Keywords Strongly dense matrix - Boolean matrix - Nonnegative matrix · Idempotent matrix · Intrinsic product - Generalized complementary basic matrix

Mathematics Subject Classification $\quad 15$ A15 $\cdot 15$ A21 15 A23 $\cdot 15 B 34$

\section{Introduction}

In [8], the authors introduced and studied so-called row-dense, column-dense, and dense matrices. A matrix is dense (row-dense, column-dense) if there are no zeros

Miroslav Fiedler was supported by RVO:67985807.

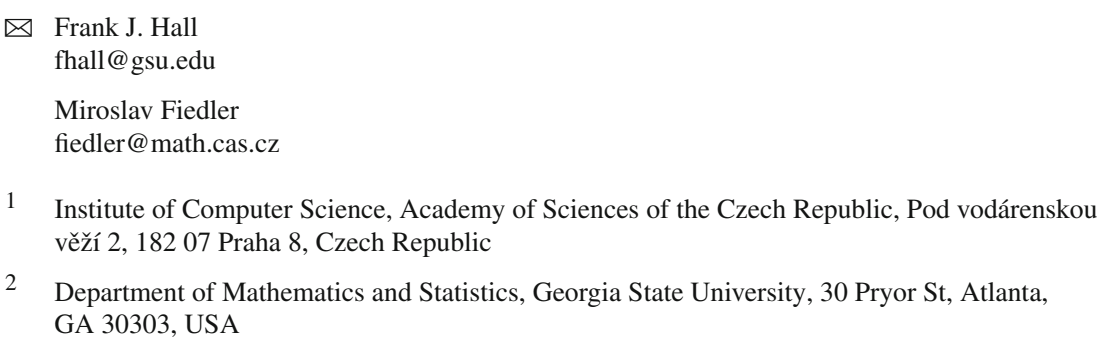


between two nonzero entries for every line (row, column) of this matrix. In this note, we show that if we specify these notions in a certain way, we obtain a class of matrices which in certain cases forms a subclass closed under multiplication. We now define a matrix to be strongly row-dense, SRD for short, if it is row-dense, does not have a zero row, and, in addition, the union of nonzero positions in any two consecutive rows is also, in a clear way, row-dense. Analogously, strongly column-dense, shortly SCD, and strongly dense, shortly SD, matrices are defined.

In the following examples, $*$ indicates a nonzero entry. The matrix

$$
A=\left[\begin{array}{llll}
0 & * & 0 & 0 \\
* & * & * & 0 \\
0 & * & * & * \\
0 & 0 & * & 0
\end{array}\right]
$$

is both SRD and SCD, hence SD. The matrix

$$
A_{1}=\left[\begin{array}{llll}
0 & 0 & 0 & 0 \\
* & 0 & 0 & 0 \\
0 & 0 & * & * \\
0 & * & 0 & 0
\end{array}\right]
$$

is row-dense, but not SRD for two reasons: $A$ contains a zero row and the union of the nonzero positions in rows 2 and 3 is not row-dense. Also, $A_{1}$ is column-dense but not $\mathrm{SCD}$, as the union of the nonzero positions in columns 1 and 2 is not column-dense. The matrix

$$
A_{2}=\left[\begin{array}{llll}
0 & * & 0 & 0 \\
* & * & 0 & 0 \\
0 & 0 & * & * \\
0 & * & 0 & 0
\end{array}\right]
$$

is $\operatorname{SRD}$ but not $\operatorname{SCD}\left(A_{2}\right.$ is not even column-dense), while

$$
A_{2}^{T}=\left[\begin{array}{llll}
0 & * & 0 & 0 \\
* & * & 0 & * \\
0 & 0 & * & 0 \\
0 & 0 & * & 0
\end{array}\right]
$$

is SCD but not SRD.

In this paper, we first give some basic properties of these matrices. In particular, we show that for nonnegative matrices and Boolean matrices, the product of conformable SRD- (SCD-, SD-) matrices is SRD (SCD, SD). We then obtain structural characterizations for the idempotent nonnegative SRD- (SCD-, SD-) matrices, as well as for the idempotent Boolean SD-matrices with a full diagonal. Connections with generalized complementary basic matrices are made. 


\section{Basic properties}

By induction with respect to the number of rows one easily observes that

Lemma 2.1 The union of nonzero positions in any non-void set of consecutive rows of an SRD-matrix is row-dense and nonzero.

We observe that two classes of matrices, namely nonnegative matrices and Boolean matrices, share common combinatorial properties with respect to density. In particular, applying Lemma 2.1, we have the following.

Theorem 2.2 In the classes of nonnegative matrices and Boolean matrices, the product of SRD nonnegative matrices which can be multiplied is SRD.

Proof For nonnegative matrices and Boolean matrices Lemma 2.1 states: If $u$ is a dense row-vector and $P$ an SRD-matrix, then $u P$ is a dense row-vector.

Let thus $A$ and $B$ be nonnegative SRD-matrices or (both) Boolean SRD-matrices which can be multiplied, and $C$ their product. Since each row of $A$ is dense, each row of $C$ is dense. To show that $C$ is SRD, let $c_{i}$ and $c_{i+1}$ be two consecutive rows of $C$. Multiply $C$ from the left by the row vector $v=(0,0, \ldots, 1,1,0, \ldots, 0)$, where the ones are in the $i$ th and $(i+1)$ st positions. We have $v C=v A B$ and $w=v A$ is dense, then $v C=w B$ is also dense, and thus $C$ is SRD.

Remark 2.3 An analogous result holds for SCD- and SD-matrices. Also, instead of assuming nonnegativity or Boolean in Theorem 2.2 one can assume that the product is intrinsic in the sense introduced in [3].

Example 2.4 Observe that square nonsingular bidiagonal matrices are SRD- as well as SCD- and SD-matrices. If they are nonnegative, it is known that their products generate the class of nonsingular totally nonnegative matrices. This fact opens many new aspects and problems for these matrices.

In the sequel, we find some simple properties of SRD-matrices useful. The following is clear.

Lemma 2.5 If an SRD-matrix has a zero column c, then either all columns before $c$ are zero columns, or all columns behind c are zero columns. Analogously, if an $S C D$-matrix has a zero row $r$, then either all rows above $r$ are zero rows, or all rows below $r$ are zero rows.

Lemma 2.6 If an SRD-matrix A has a block diagonal form

$$
A=\left[\begin{array}{cccc}
D_{1} & 0 & \cdots & 0 \\
0 & D_{2} & \cdots & 0 \\
\vdots & \vdots & \ddots & \vdots \\
0 & 0 & \cdots & D_{k}
\end{array}\right],
$$

where $D_{i}$ are square positive matrices of rank one, then for every permutation matrix $P$ for which $P A P^{T}$ is $S R D$, the matrix $P A P^{T}$ also has such form with the same $k$. 
Proof Every diagonal block $D_{i}$ can be written as $X_{i} Y_{i}^{T}$, where $X_{i}$ and $Y_{i}$ are positive column vectors of the same mutual dimension. Thus $A=X Y^{T}$, where

$$
X=\left[\begin{array}{cccc}
X_{1} & 0 & \cdots & 0 \\
0 & X_{2} & \cdots & 0 \\
\vdots & \vdots & \ddots & \vdots \\
0 & 0 & \cdots & X_{k}
\end{array}\right], \quad Y=\left[\begin{array}{cccc}
Y_{1} & 0 & \cdots & 0 \\
0 & Y_{2} & \cdots & 0 \\
\vdots & \vdots & \ddots & \vdots \\
0 & 0 & \cdots & Y_{k}
\end{array}\right]
$$

are equally partitioned.

Then $Y^{T} P^{T}$ is a row-dense matrix. Indeed, suppose that in the $m$ th row of $Y^{T} P^{T}$ there is a zero between nonzeros. There is a row in $P X$, say row $q$, which has a single nonzero entry in the $m$ th column. It follows that in $P A P^{T}$ the $q$ th row is not dense, a contradiction. This fact implies that the groups of consecutive indices in $Y^{T} P^{T}$ are by the permutation $P$ either only permuted, or the groups as a whole are permuted themselves. The same happens with $P X$. Altogether, $P A P^{T}$ has a block form, with $k$ blocks positive and of rank one.

Remark 2.7 We call a square positive matrix of rank one basic. We also call a direct sum of matrices complete if it does not have a zero row. In this sense, matrices (1) and $P A P^{T}$ are complete direct sums of basic matrices.

\section{Idempotents}

In this section, we investigate the structure of SRD-matrices. Since nonnegative matrices form a multiplicative semigroup, it is natural to study idempotent SRD-matrices first. For nonnegative matrices, the basic result of Flor [9], as given in the book [2], can be used for SRD-matrices.

Theorem 3.1 (Flor) Let $E$ be a nonnegative idempotent matrix of rank $k$. Then there exists a permutation matrix $P$ such that

$$
P E P^{T}=\left[\begin{array}{cccc}
J & J U & 0 & 0 \\
0 & 0 & 0 & 0 \\
V J & V J U & 0 & 0 \\
0 & 0 & 0 & 0
\end{array}\right], \quad J=\left[\begin{array}{cccc}
J_{1} & 0 & \cdots & 0 \\
0 & J_{2} & \cdots & 0 \\
\vdots & \vdots & \ddots & \vdots \\
0 & 0 & \cdots & J_{k}
\end{array}\right],
$$

where $J_{i}$ are idempotent matrices of rank one, i.e., $J_{i}=x^{(i)} y^{(i) T}$, where $x^{(i)}, y^{(i)}$ are positive column vectors satisfying $y^{(i) T} x^{(i)}=1$, and $U$ and $V$ are nonnegative matrices of appropriate sizes.

Conversely, every square matrix of the form (2), with $U$ and $V$ arbitrary nonnegative matrices of appropriate sizes, is idempotent and of rank $k$.

If specified for matrices without zero rows, Flor's theorem states 
Lemma 3.2 Let $E$ be a nonnegative idempotent matrix of rank $k$ without zero rows. Then there exists a permutation matrix $P$ such that

$$
P E P^{T}=\left[\begin{array}{cc}
J & 0 \\
V J & 0
\end{array}\right], \quad J=\left[\begin{array}{cccc}
J_{1} & 0 & \cdots & 0 \\
0 & J_{2} & \cdots & 0 \\
\vdots & \vdots & \ddots & \vdots \\
0 & 0 & \cdots & J_{k}
\end{array}\right],
$$

where $J_{i}$ are idempotent matrices of rank one and $V$ is a nonnegative matrix of appropriate size.

Conversely, every square matrix of the form (3), with $V$ an arbitrary nonnegative matrix of appropriate size, is idempotent and of rank $k$.

Remark 3.3 If $E$ does not have a zero row, then the same holds for $V$.

Theorem 3.4 A nonnegative matrix $E$ is an idempotent SRD-matrix of rank $r$ if and only if it has the symmetrically partitioned form

$$
E=\left[\begin{array}{lll}
0 & C Y^{T} & 0 \\
0 & X Y^{T} & 0 \\
0 & D Y^{T} & 0
\end{array}\right]
$$

where

$$
X=\left[\begin{array}{cccc}
X_{1} & 0 & \cdots & 0 \\
0 & X_{2} & \cdots & 0 \\
\vdots & \vdots & \ddots & \vdots \\
0 & 0 & \cdots & X_{r}
\end{array}\right], \quad Y=\left[\begin{array}{cccc}
Y_{1} & 0 & \cdots & 0 \\
0 & Y_{2} & \cdots & 0 \\
\vdots & \vdots & \ddots & \vdots \\
0 & 0 & \cdots & Y_{r}
\end{array}\right]
$$

are equally partitioned, $X_{i}$ and $Y_{j}$ are positive column vectors satisfying $Y_{i}^{T} X_{i}=1$, $i=1, \ldots, r$, and $C$ and $D$ nonnegative matrices such that

$$
\left[\begin{array}{l}
C \\
I_{r} \\
D
\end{array}\right]
$$

is an SRD-matrix.

Proof $(\Leftarrow)$ The SRD property of $E$ follows from Theorem 2.2 since $E$ is the product of the SRD-matrices

$$
\left[\begin{array}{lll}
0 & C & 0 \\
0 & X & 0 \\
0 & D & 0
\end{array}\right] \text { and }\left[\begin{array}{ccc}
I & 0 & 0 \\
0 & Y^{T} & 0 \\
0 & 0 & I
\end{array}\right] .
$$


$(\Rightarrow)$ Let $E$ be an idempotent SRD-matrix of rank $r$. By Lemma 2.5, the zero columns in $E$ can be at most some first columns and at most some last columns. By Lemma 3.2, there exists a permutation matrix $P$ such that

$$
P E P^{T}=\left[\begin{array}{cc}
J & 0 \\
V J & 0
\end{array}\right], \quad J=\left[\begin{array}{cccc}
J_{1} & 0 & \cdots & 0 \\
0 & J_{2} & \cdots & 0 \\
\vdots & \vdots & \ddots & \vdots \\
0 & 0 & \cdots & J_{r}
\end{array}\right],
$$

where $J_{i}$ are idempotent matrices of rank one. Since $P$ preserves the diagonal of $E$, $E$ has the block symmetrically partitioned form

$$
E=\left[\begin{array}{lll}
0 & E_{1} & 0 \\
0 & E_{2} & 0 \\
0 & E_{3} & 0
\end{array}\right],
$$

where $E_{2}$ is a permuted matrix of $J$. Since $E$ is an SRD-matrix, the diagonal block $E_{2}$ is, by Lemma 2.6, also a matrix of the form $X Y^{T}$ with possibly permuted rows of $X$ and correspondingly permuted columns of $Y^{T}$. The condition $Y^{T} X=I$ is also satisfied. Since $E$ is idempotent, $E_{1}=E_{1} E_{2}=E_{1} X Y^{T}$, the matrix $E_{1} X$ plays the role of $C$ and $E_{3} X$ plays the role of $D$. It remains to show that the matrix $\left[\begin{array}{lll}C & I_{r} & D\end{array}\right]^{T}$ in (4) is an SRD-matrix.

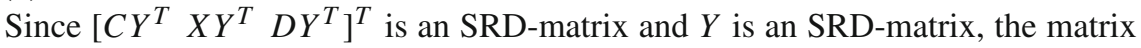
$\left[\begin{array}{lll}C Y^{T} Y & X Y^{T} Y & D Y^{T} Y\end{array}\right]^{T}$ is an SRD-matrix. Since $Y^{T} Y$ is a diagonal matrix with positive diagonal entries, the matrix $\left[\begin{array}{lll}C & X & D\end{array}\right]^{T}$ is an SRD-matrix, and thus also the matrix $\left[\begin{array}{lll}C & I_{r} & D\end{array}\right]^{T}$ is $\mathrm{SRD}$.

Corollary 3.5 A complete characterization of idempotent nonnegative SCD-matrices is obtained by transposition of the result in Theorem 3.4.

Recalling definitions in Remark 2.7, we also have

Corollary 3.6 A nonnegative matrix is an idempotent SD-matrix if and only if it is a complete direct sum of idempotent basic matrices. The number of the basic matrices is then the rank of the direct sum.

Let us turn now to idempotents in the class of Boolean matrices. We start with idempotent SD-matrices. The following is clear.

Observation 3.7 A Boolean idempotent upper triangular SD-matrix $U$ is a matrix which is formed by the upper triangular part of a direct sum $B_{U}$ of unit (i.e. full of ones) diagonal blocks. Similarly, a Boolean idempotent lower triangular SD-matrix $L$ is a matrix which is formed by the lower triangular part of a direct sum $B_{L}$ of unit diagonal blocks. (Of course, the diagonal is considered included to the triangular part.) 
For example, if

$$
B_{U}=\left[\begin{array}{lllll}
1 & 1 & 0 & 0 & 0 \\
1 & 1 & 0 & 0 & 0 \\
0 & 0 & 1 & 1 & 1 \\
0 & 0 & 1 & 1 & 1 \\
0 & 0 & 1 & 1 & 1
\end{array}\right]
$$

then

$$
U=\left[\begin{array}{lllll}
1 & 1 & 0 & 0 & 0 \\
0 & 1 & 0 & 0 & 0 \\
0 & 0 & 1 & 1 & 1 \\
0 & 0 & 0 & 1 & 1 \\
0 & 0 & 0 & 0 & 1
\end{array}\right]
$$

is a Boolean idempotent upper triangular SD-matrix.

We can now completely characterize Boolean idempotent SD-matrices with a full diagonal.

Remark 3.8 The example

$$
\left[\begin{array}{lll}
0 & 1 & 0 \\
0 & 1 & 0 \\
1 & 1 & 1
\end{array}\right]
$$

shows that not every idempotent SD-matrix has a full diagonal.

Theorem 3.9 A Boolean SD-matrix with a full diagonal is idempotent if and only if both its upper and lower parts are idempotent Boolean SD-matrices.

Proof Let a Boolean $n \times n$ SD-matrix $A$ with a full diagonal be written as $A=$ $I+L_{0}+U_{0}$, where $I$ is the identity matrix, $L_{0}$ is the strict lower, and $U_{0}$ is the strict upper triangular part of $A$.

Suppose first that both matrices $U=I+U_{0}$ and $L=I+L_{0}$ are idempotent SD-matrices. To prove that $A$ is idempotent, it suffices to show that both Boolean products $L U$ and $U L$ are majorized by $I+L_{0}+U_{0}$.

In addition to the notation $B_{U}$ and $B_{L}$ from Observation 3.7 we use the notation $L_{C}$ for the complete Boolean lower triangular matrix and $U_{C}$ for the complete Boolean upper triangular matrix with ones in the whole part. We thus have the chain of inequalities in the Boolean sense

$$
L U \leqslant L_{C} U=L_{C}+B_{U}=L_{C}+U,
$$

which implies that the upper triangular part of $L U$ is majorized by the upper triangular part of $A$. Also,

$$
L U \leqslant L U_{C}=B_{L} U_{C}=B_{L}+U_{C}=L+U_{C}
$$


which implies that the lower triangular part of $L U$ is majorized by the lower triangular part of $A$. Altogether, $L U$ is majorized by $A$. Analogously, one shows that $U L$ is majorized by $A$, so that $A$ is idempotent.

To prove the converse part, suppose that a Boolean SD-matrix $A$ with a full diagonal is idempotent. Write as before $A=I+L_{0}+U_{0}$. For $L=I+L_{0}, A=L+U_{0}$ so that

$$
L^{2} \leqslant\left(L+U_{0}\right)^{2}=L+U_{0}
$$

Thus $L^{2}$ is majorized by $L+U_{0}$, and as well by $L$, since $U_{0}$ is strict upper triangular. Next, since

$$
L^{2}=L\left(I+L_{0}\right)=L+L L_{0} \geqslant L,
$$

$L$ is majorized by $L^{2}$. Altogether, $L^{2}=L$. Analogously, $U=I+U_{0}$ is idempotent. Finally, since $A$ is an SD-matrix, $L$ and $U$ are SD-matrices.

Remark 3.10 We mention the interesting paper [1] by Beasley et al., where it is proved that a Boolean matrix is idempotent if and only if it can represented as a sum of line and rectangular parts of a certain specific structure. However, our Theorem 3.9 specifically characterizes when a Boolean SD-matrix with a full diagonal is idempotent. It is not clear how the structure in [1] would apply to SD-matrices.

\section{Connections with GCB-matrices}

Let $A_{1}, A_{2}, \ldots, A_{s}$ be real matrices of respective orders $k_{1}, k_{2}, \ldots, k_{s}, k_{i} \geqslant 2$ for all $i$. Denote $n=\sum_{i=1}^{s} k_{i}-s+1$ and form the block diagonal matrices $G_{1}, G_{2}, \ldots, G_{s}$ as follows:

$$
\begin{aligned}
G_{1} & =\left[\begin{array}{cc}
A_{1} & 0 \\
0 & I_{n-k_{1}}
\end{array}\right], \quad G_{2}=\left[\begin{array}{ccc}
I_{k_{1}-1} & 0 & 0 \\
0 & A_{2} & 0 \\
0 & 0 & I_{n-k_{1}-k_{2}+1}
\end{array}\right], \\
G_{s-1} & =\left[\begin{array}{ccc}
I_{n-k_{s-1}-k_{s}+1} & 0 & 0 \\
0 & A_{s-1} & 0 \\
0 & 0 & I_{k_{s}-1}
\end{array}\right], \quad G_{s}=\left[\begin{array}{cc}
I_{n-k_{s}} & 0 \\
0 & A_{s}
\end{array}\right] .
\end{aligned}
$$

Then, for any permutation $\left(i_{1}, i_{2}, \ldots, i_{s}\right)$ of $(1,2, \ldots, s)$, we can consider the product

$$
G_{i_{1}} G_{i_{2}} \cdots G_{i_{s}}
$$

We call products of this form generalized complementary basic matrices, GCBmatrices for short. We use the notation $\prod G_{k}$ for these general products. The diagonal blocks $A_{k}$ are called distinguished blocks and $G_{k}$ are called generators of $\prod G_{k}$. GCBmatrices have many striking properties such as permanental, graph theoretic, spectral, and inheritance properties (see for example [5-7]). 
The upper-left and lower-right corner entries of each matrix $A_{k}$ will be called intermediate and will be assumed to be nonzero.

Lemma 4.1 Suppose $n>k>1$. Let

$$
A_{0}=\left[\begin{array}{ccc}
a_{11} & \cdots & a_{1 k} \\
\vdots & \ddots & \vdots \\
a_{k 1} & \cdots & a_{k k}
\end{array}\right]
$$

be a $k \times k$ matrix, and

$$
B_{0}=\left[\begin{array}{ccc}
b_{k k} & \cdots & b_{k n} \\
\vdots & \ddots & \vdots \\
b_{n k} & \cdots & b_{n n}
\end{array}\right]
$$

be an $(n-k+1) \times(n-k+1)$ matrix (the sum of orders of $A_{0}$ and $B_{0}$ thus exceeds $n$ by one). Then, for the $n \times n$ matrices

$$
A=\left[\begin{array}{cc}
A_{0} & 0 \\
0 & I_{n-k}
\end{array}\right] \quad \text { and } \quad B=\left[\begin{array}{cc}
I_{k-1} & 0 \\
0 & B_{0}
\end{array}\right] \text {, }
$$

the product $A B$ has the explicit form

$$
A B=\left[\begin{array}{cccccc}
a_{11} & \cdots & a_{1, k-1} & a_{1 k} b_{k k} & \cdots & a_{1 k} b_{k n} \\
a_{21} & \cdots & a_{2, k-1} & a_{2 k} b_{k k} & \cdots & a_{2 k} b_{k n} \\
\vdots & \ddots & \vdots & \vdots & \ddots & \vdots \\
a_{k 1} & \cdots & a_{k, k-1} & a_{k k} b_{k k} & \cdots & a_{k k} b_{k n} \\
0 & \cdots & 0 & b_{k+1, k} & \cdots & b_{k+1, n} \\
\vdots & \ddots & \vdots & \vdots & \ddots & \vdots \\
0 & \cdots & 0 & b_{n k} & \cdots & b_{n n}
\end{array}\right] .
$$

Theorem 4.2 Suppose that GCB-matrices $A$ and $B$ are as in Lemma 4.1, where $a_{11}, a_{k k}, b_{k k}, b_{n n}$ are nonzero. If both $A_{0}$ and $B_{0}$ are SRD (SCD), then both $A B$ and $B A$ are $S R D(S C D)$.

Proof The proof of the assertion for $A B$ follows by examining the product in (5); the proof for $B A$ is similar.

Corollary 4.3 Assume that all intermediate entries in matrices $A_{k}$ are nonzero. If each $A_{k}$ is $S R D(S C D)$, then the generalized complementary basic matrix $\prod G_{k}$ is $S R D(S C D)$, the order of factors does not matter.

Proof Let $A_{k}, k=1, \ldots, s$, be SRD; the proof for SCD is similar. We proceed by induction. If $s=2$, the result follows from Theorem 4.2. Suppose that $s>2$ and that the result holds for $s-1$ matrices. Observe that matrices $G_{i}$ and $G_{k}$ commute if 
$|i-k|>1$. This means that if 1 is before 2 in the permutation $\left(i_{1}, i_{2}, \ldots, i_{s}\right)$, we can move $G_{1}$ into the first position without changing the product. The product $\Pi$ of the remaining $s-1$ matrices $G_{k}$ has the form

$$
\left[\begin{array}{cc}
I_{k_{1}-1} & 0 \\
0 & B_{0}
\end{array}\right] \text {. }
$$

By the induction hypothesis, $B_{0}$ is SRD. Hence, by Theorem $4.2, \prod G_{k}=G_{1} \Pi$ is SRD.

If 1 is behind 2 in the permutation, we can move $G_{1}$ into the last position and then we have a $B A$ product as in Theorem 4.2.

Strongly dense matrices deserve further exploration. This investigation can include minimum ranks of the corresponding sign pattern matrices, as was done in [4] for the dense Alternating Sign Matrices. Connections to the interesting paper [1] might also be made.

Acknowledgments The authors thank the referee for many useful comments.

\section{References}

1. Beasley, L.B., Guterman, A.E., Kang, K.-T., Song, S.-Z.: Idempotent Boolean matrices and majorization. J. Math. Sci. (N.Y.) 152(4), 456-468 (2008)

2. Berman, A., Plemmons, R.J.: Nonnegative Matrices in the Mathematical Sciences. Classics in Applied Mathematics, vol. 9. SIAM, Philadelphia (1994)

3. Fiedler, M.: Intrinsic products and factorizations of matrices. Linear Algebra Appl. 428(1), 5-13 (2008)

4. Fiedler, M., Gao, W., Hall, F.J., Jing, G., Li, Z., Stroev, M.: Ranks of dense alternating sign matrices and their sign patterns. Linear Algebra Appl. 471, 109-121 (2015)

5. Fiedler, M., Hall, F.J.: A note on permanents and generalized complementary basic matrices. Linear Algebra Appl. 436(9), 3553-3561 (2012)

6. Fiedler, M., Hall, F.J.: Some graph theoretic properties of generalized complementary basic matrices. Linear Algebra Appl. 438(8), 3365-3374 (2013)

7. Fiedler, M., Hall, F.J.: Combinatorial aspects of generalized complementary basic matrices. Cent. Eur. J. Math. 11(12), 2186-2196 (2013)

8. Fiedler, M., Hall, F.J., Stroev, M.: Dense alternating sign matrices and extensions. Linear Algebra Appl. 444, 219-226 (2014)

9. Flor, P.: On groups of non-negative matrices. Compositio Math. 21(4), 376-382 (1969) 